\title{
Cutting Costs in Inguinal Hernia Surgery: Laparoscopic or Ambulatory (or 1-day) Open Hernia (Lichtenstein) Repair?
}

\author{
Andrea Cariati • Elisa Piromalli
}

Received: 24 January 2012 / Accepted: 5 June 2012 / Published online: 17 June 2012

(C) Association of Surgeons of India 2012

Recently in the Indian Journal of Surgery [1], an interesting article on the various techniques used for the inguinal hernia repair was published. Data reported from Western countries revealed some differences. In particular, a study conducted in Boston, USA, reported that laparoscopic approach could be a cost-effective treatment option for inguinal hernia repair [2]. In order to compare these differences, further considerations are necessary. In fact, laparoscopic hernia repair has been widely recommended in the past 20 years, but this procedure, in Europe, is nearly $€ 400$ more expensive than ambulatory (or 1-day) anterior mesh (Lichtenstein) herniorrhaphy [3]. Every year in the USA, the UK, and Italy nearly 800000 [4], 80000, and 150000 inguinal herniorrhaphies, respectively, are performed.

If all of these operations are done laparoscopically, the annual hospital costs of each state would be $€ 320$ million (USA), $€ 32$ million (UK), or $€ 60$ million (Italy) higher, respectively.

In conclusion, if all these techniques, in the hands of expert surgeons, have the same rates of recurrence and complications, in order to reduce and contain the hospitalization costs for hernia surgery, laparoscopic herniorrhaphy should be used only in very selected cases as plurirecurrent inguinal hernias. Actually, the preferred operation for inguinal hernia should be ambulatory (or 1-day) anterior mesh (Lichtenstein) herniorrhaphy.

\section{References}

1. Anand A, Sinha PA, Kittappa K, Mulchandani MH, Debrah S, Brookstein R (2011) Review of inguinal hernia repairs by various surgical techniques in a district general hospital in the UK. Indian J Surg 73:13-18

2. Stylopoulos N, Gazelle GS, Rattner DW (2003) A cost-utility analysis of treatment options for inguinal hernia in 1.513.008 adult patients. Surg Endosc 17:180-189

3. Medical Research Council Laparoscopic Groin Hernia Trial Group (2001) Cost-utility analysis of open versus laparoscopic groin hernia repair: results from a multicentre randomized clinical trial. Br J Surg 88:653-661

4. Rutkow IM (2003) Demographic and socioeconomic aspects of hernia repair in the United States in 2003. Surg Clin North Am 83:1045-1051

A. Cariati $(\bowtie) \cdot$ E. Piromalli

San Martino and IST Hospital,

Via Fratelli Coda 67/5 a,

16166, Genoa, Italy

e-mail: andrea.cariati@libero.it 